PROCEEDINGS OF THE

AMERICAN MATHEMATICAL SOCIETY

Volume 131, Number 7 , Pages 1981-1988

S 0002-9939(03)06813-8

Article electronically published on February 20, 2003

\title{
PERTURBATION OF WIGNER MATRICES AND A CONJECTURE
}

\author{
MARK FANNES AND DÉNES PETZ
}

(Communicated by David R. Larson)

\begin{abstract}
Let $H_{0}$ be an arbitrary self-adjoint $n \times n$ matrix and $H(n)$ be an $n \times n$ (random) Wigner matrix. We show that $t \mapsto \operatorname{Tr} \exp \left(H(n)-\mathrm{i} t H_{0}\right)$ is positive definite in the average. This partially answers a long-standing conjecture. On the basis of asymptotic freeness our result implies that $t \mapsto \tau(\exp (a-\mathrm{i} t b))$ is positive definite whenever the noncommutative random variables $a$ and $b$ are in free relation, with $a$ semicircular.
\end{abstract}

\section{INTRODUCTION}

Let $H$ and $H_{0}$ be self-adjoint $n \times n$ matrices. It is a widely known conjecture [1] 6. 9, 10 that the function

$$
t \mapsto \operatorname{Tr} \mathrm{e}^{H-\mathrm{i} t H_{0}}
$$

is positive definite on $\mathbb{R}$. This means that there exists a measure $\mu$ on $\mathbb{R}$ whose Fourier transform is the above function:

$$
\operatorname{Tr} \mathrm{e}^{H-\mathrm{i} t H_{0}}=\frac{1}{\sqrt{2 \pi}} \int \mathrm{e}^{-\mathrm{i} t x} d \mu(x) \quad(t \in \mathbb{R}) .
$$

We shall call $\mu$ the Bochner measure of the function (1), if it really exists. If this is the case, then $\mu$ depends both on the spectra of the two matrices and on the relative position of their eigenvectors. The function (1), and especially its derivatives at $t=0$, define important quantities in quantum statistical mechanics. Proving positive definiteness would lead to interesting relations among them. The $n$th derivative at $t=0$ of (1) is, up to a factor $\mathrm{i}^{n}$, given by

$$
a_{n}=\int_{\substack{0 \leq t_{1}, 0 \leq t_{2}, \ldots, 0 \leq t_{n-1} \\ t_{1}+t_{2}+\cdots+t_{n-1} \leq 1}} d t_{1} \cdots d t_{n-1} \operatorname{Tr} \mathrm{e}^{\left(1-t_{1}-\cdots-t_{n-1}\right) H} H_{0} \mathrm{e}^{t_{1} H} H_{0} \cdots \mathrm{e}^{t_{n-1} H} H_{0} .
$$

Positive definiteness of (1) is then equivalent to

$$
\operatorname{det}\left(\left[a_{i+j}\right]_{i, j=0,1, \ldots, n}\right) \geq 0 \quad(n \in \mathbb{N}) .
$$

Received by the editors July 6, 2001.

2000 Mathematics Subject Classification. Primary 15A15, 15A62, 46L54.

Key words and phrases. Bessis-Moussa-Vilani conjecture, Gaussian random matrix, Wigner theorem, positive definite function, free random variables, semicircular element.

The second author was partially supported by OTKA T 032662. 
The aim of this paper is to show that the conjecture holds in the average for some random choices of matrices. More precisely, when the conjecture is true, then for any choice of self-adjoint $n \times n$ random matrix $H_{n}$ the function

$$
F(t):=\frac{1}{n} \mathbb{E}\left(\operatorname{Tr} \mathrm{e}^{H_{n}-\mathrm{i} t H_{0}}\right)
$$

is positive definite (for any $H_{0}$ ). We deal with the particular case in which $H_{n}$ has independent Gaussian entries. We shall give rather explicitly the measure $\mu$ whose Fourier transform is $F(t)$. It depends only on the spectrum of the matrix $H_{0}$ since the eigenvectors of $H_{n}$ have a rotationally invariant distribution. It turns out that the support of $\mu$ is the convex hull of the spectrum of $H_{0}$.

The result for matrices has a consequence for free random variables $a$ and $b$ when $a$ is semicircular. The pair $(a, b)$ has a random matrix model consisting of a Wigner matrix and a diagonal matrix. From the matrix result we can conclude the positive definiteness of $\tau\left(e^{a-\mathrm{i} t b}\right)$ letting the matrix size go to infinity.

\section{$n \times n$ MATRICES}

An $n \times n$ complex self-adjoint random matrix $H(n)$ is called a Wigner matrix if

(i) $\left\{\operatorname{Re} H_{i j}(n) \mid 1 \leq i \leq j \leq n\right\} \cup\left\{\operatorname{Im} H_{i j}(n) \mid 1 \leq i<j \leq n\right\}$ is an independent family of Gaussian random variables, and

(ii) $\mathbb{E}\left(H_{i j}(n)\right)=0$ for $1 \leq i \leq j \leq n, \mathbb{E}\left(H_{i i}(n)^{2}\right)=1 / n$ for $1 \leq i \leq n$, and $\mathbb{E}\left(\left(\operatorname{Re} H_{i j}(n)\right)^{2}\right)=\mathbb{E}\left(\left(\operatorname{Im} H_{i j}(n)\right)^{2}\right)=1 / 2 n$ for $1 \leq i<j \leq n$.

The Wigner matrix is standard because $\tau_{n}(H(n))=0$ and $\tau_{n}\left(H(n)^{2}\right)=1$, where

$$
\tau_{n}:=\frac{1}{n} \mathbb{E} \circ \operatorname{Tr}_{n}
$$

Our aim is to prove the following:

Theorem. Let $H_{0}$ be a fixed self-adjoint matrix and $H(n)$ a standard Wigner matrix. Then the function $t \mapsto \tau_{n}\left(\exp \left(H(n)-i t H_{0}\right)\right)$ is positive definite. Its corresponding Bochner measure is the sum of an atomic and an absolutely continuous part. The atomic part is concentrated at the eigenvalues of $H_{0}$ and the support of the absolutely continuous part coincides with the convex hull of the spectrum of $H_{0}$.

In the following, we first give the probability density of the $n \times n$ random matrix $H_{0}+H(n)$. Then, we assume that $H_{0}$ has the eigenvalues $d_{1}<d_{2}<\cdots<d_{n}$ and compute the above function explicitly in terms of these eigenvalues (cf. [4] or [7]).

The probability density function of $H(n)$ is

$$
C_{n} \exp \left(-\frac{n}{2} \operatorname{Tr} A^{2}\right) \quad \text { with } \quad C_{n}=2^{-n / 2}\left(\frac{\pi}{n}\right)^{-n^{2} / 2}
$$

with respect to the Lebesgue measure

$$
d A:=\prod_{i=1}^{n} d A_{i i} \prod_{i<j} d\left(\operatorname{Re} A_{i j}\right) d\left(\operatorname{Im} A_{i j}\right) \quad \text { on } \quad M_{n}(\mathbb{C})^{s a} \cong \mathbb{R}^{n^{2}} .
$$

The density of $H_{0}+H(n)$ with respect to the measure (2) is

$$
C\left(H_{0}\right) \exp \left(-\frac{n}{2} \operatorname{Tr} A^{2}+n \operatorname{Tr} H_{0} A\right) .
$$


We have

$$
\begin{aligned}
F: & =\tau_{n}\left(\exp \left(H(n)+H_{0}\right)\right) \\
& =\frac{C\left(H_{0}\right)}{n} \int \operatorname{Tr} \mathrm{e}^{A} \exp \left(-\frac{n}{2} \operatorname{Tr} A^{2}+n \operatorname{Tr} H_{0} A\right) d A
\end{aligned}
$$

Since the measure is unitarily invariant, we can first integrate with respect to the Haar probability $d U$ over $\mathcal{U}(n)$ :

$$
F=\frac{C\left(H_{0}\right)}{n} \int \operatorname{Tr}^{A} \exp \left(-\frac{n}{2} \operatorname{Tr} A^{2}\right)\left(\int \exp \left(n \operatorname{Tr} U A U^{*} H_{0}\right) d U\right) d A .
$$

In this way, we can use the integral formula (see [8], A.5, and also [2], Theorem 7.24, for a more general formula attributed to Harish-Chandra)

$$
\int \exp \left(\operatorname{Tr} U A U^{*} B\right) d U=\frac{\operatorname{det}\left[\exp \left(\lambda_{i} \rho_{j}\right)\right]}{\Delta(\lambda) \Delta(\rho)}
$$

where $A, B$ are $n \times n$ self-adjoint matrices, the $\lambda_{i}$ 's are the eigenvalues of $A$, the $\rho_{j}$ 's are those of $B$, and

$$
\Delta(\lambda) \equiv \Delta\left(\lambda_{1}, \lambda_{2}, \ldots, \lambda_{n}\right)=: \prod_{i<j}\left(\lambda_{i}-\lambda_{j}\right) .
$$

Since $d A=C \Delta(\lambda)^{2} d \lambda$, we can calculate as follows:

$$
\begin{aligned}
F & =\frac{C\left(H_{0}\right)}{n} \int \operatorname{Tr} \mathrm{e}^{A} \exp \left(-\frac{n}{2} \operatorname{Tr} A^{2}\right) \frac{\operatorname{det}\left[\exp \left(n \lambda_{i} d_{j}\right)\right]}{\Delta(\lambda) \Delta(d)} d A \\
& =\frac{C^{\prime}\left(H_{0}\right)}{n} \int\left(\sum_{j} \mathrm{e}^{\lambda_{j}}\right) \exp \left(-\frac{n}{2} \sum_{i} \lambda_{i}^{2}\right) \Delta(\lambda)^{2} \frac{\operatorname{det}\left[\exp \left(n \lambda_{i} d_{j}\right)\right]}{\Delta(\lambda) \Delta(d)} d \lambda \\
& =\frac{C^{\prime}\left(H_{0}\right)}{n \Delta(d)} \int\left(\sum_{j} \mathrm{e}^{\lambda_{j}}\right) \exp \left(-\frac{n}{2} \sum_{i} \lambda_{i}^{2}\right) \Delta(\lambda) \operatorname{det}\left[\exp \left(n \lambda_{i} d_{j}\right)\right] d \lambda
\end{aligned}
$$

where the latter integrals are over $\mathbb{R}_{\leq}^{n}:=\left\{\left(x_{1}, x_{2}, \ldots, x_{n}\right) \in \mathbb{R}^{n} \mid x_{1} \leq x_{2} \leq \cdots \leq\right.$ $\left.x_{n}\right\}$. Integration over $\mathbb{R}^{n}$ gives a factor $n$ !. We expand the determinant by summing over all permutations $\sigma$ of $\{1,2, \ldots, n\}$ :

$$
\frac{C^{\prime}\left(H_{0}\right)}{n ! n \Delta(d)} \sum_{\sigma} \int\left(\sum_{j} \mathrm{e}^{\lambda_{j}}\right) \exp \left(-\frac{n}{2} \sum_{i} \lambda_{i}^{2}\right) \Delta(\lambda)(-1)^{|\sigma|} \prod_{i} \exp \left(n \lambda_{i} d_{\sigma(i)}\right) d \lambda
$$

and all summands are the same, due to the fact that $\Delta$ changes sign when two of its arguments are exchanged. Hence we arrive at

$$
F=\frac{C^{\prime}\left(H_{0}\right)}{n \Delta(d)} \int\left(\sum_{j} \mathrm{e}^{\lambda_{j}}\right) \exp \left(-\frac{n}{2} \sum_{i} \lambda_{i}^{2}+n \sum_{i} \lambda_{i} d_{i}\right) \Delta(\lambda) d \lambda .
$$

Now we proceed by means of the integral

$$
\int \exp \left(-\frac{n}{2} \sum_{i}\left(\lambda_{i}-a_{i}\right)^{2}\right) \Delta(\lambda) d \lambda=C(n) \Delta(a)
$$


and conclude that

$$
\begin{aligned}
F= & \left.\frac{C^{\prime \prime}\left(H_{0}\right)}{n} \exp \left(\frac{n}{2} \sum_{i} d_{i}^{2}\right)\right) \\
& \times \sum_{j} \mathrm{e}^{d_{j}} \frac{\Delta\left(d_{1}, \ldots, d_{j-1}, d_{j}+1 / n, d_{j+1}, \ldots, d_{n}\right)}{\Delta\left(d_{1}, d_{2}, \ldots, d_{n}\right)} \\
= & \frac{1}{n} \exp \left(\frac{1}{2 n}\right) \sum_{j} \mathrm{e}^{d_{j}} \prod_{i \neq j} \frac{d_{i}-d_{j}-1 / n}{d_{i}-d_{j}} \\
= & \frac{1}{n} \exp \left(\frac{1}{2 n}\right) \sum_{j} \mathrm{e}^{d_{j}} \prod_{i \neq j}\left(1-\frac{1}{n\left(d_{i}-d_{j}\right)}\right) .
\end{aligned}
$$

Using analytic continuation, we replace $d_{j}$ by $-\mathrm{i} t d_{j}$ and obtain

$$
\tau_{n}\left(\exp \left(H(n)-\mathrm{i} t H_{0}\right)\right)=\frac{1}{n} \exp \left(\frac{1}{2 n}\right) \sum_{j} \mathrm{e}^{-\mathrm{i} d_{j} t} \prod_{i \neq j}\left(1+\frac{1}{\mathrm{i} n t\left(d_{i}-d_{j}\right)}\right) .
$$

Our theorem states that this is a positive definite function of $t$. We shall explicitly obtain the inverse Fourier transform of $\tau_{n}\left(\exp \left(H(n)-\mathrm{i} t H_{0}\right)\right)$ and show that it is a positive measure supported on $\left[d_{1}, d_{n}\right]$. So, we compute, given an $n$-tuple $\mathbf{d}=\left\{d_{1}, d_{2}, \ldots, d_{n}\right\}$ with $d_{1}<d_{2}<\cdots<d_{n}$, the inverse Fourier transform of the function

$$
F(\mathbf{d} ; t):=\sum_{j=1}^{n} \mathrm{e}^{-\mathrm{i} d_{j} t} \prod_{\substack{k=1 \\ k \neq j}}^{n}\left(1+\frac{1}{\mathrm{i} \operatorname{tn}\left(d_{k}-d_{j}\right)}\right) .
$$

The computation is rather direct, expanding the product in (3) in inverse powers of $t$

$$
F(\mathbf{d} ; t)=\sum_{m=0}^{n}\left(\frac{\mathrm{i}}{n t}\right)^{m} \sum_{j=1}^{n} \sum_{\substack{k_{1}, k_{2}, \ldots, k_{m} \\ k_{1}<k_{2}<\ldots<k_{m} \\ k_{\ell} \neq j}} \frac{\mathrm{e}^{-\mathrm{i} d_{j} t}}{\left(d_{j}-d_{k_{1}}\right) \cdots\left(d_{j}-d_{k_{m}}\right)} .
$$

Only the singularity at $t=0$ in (4) is apparent and we shall first remove it by using for each $m$-term the Taylor expansion of the function $d \mapsto \exp (-\mathrm{i} d t)$ around the point $d_{1}$ up to order $m$ :

$$
\begin{aligned}
& \mathrm{e}^{-\mathrm{i} d t}=\mathrm{e}^{-\mathrm{i} d_{1} t}+(-\mathrm{i} t)\left(d-d_{1}\right) \mathrm{e}^{-\mathrm{i} d_{1} t} \\
&+\cdots+\frac{1}{(m-1) !}(-\mathrm{i} t)^{m-1}\left(d-d_{1}\right)^{m-1} \mathrm{e}^{-\mathrm{i} d_{1} t} \\
&+(-\mathrm{i} t)^{m} \frac{1}{(m-1) !} \int_{d_{1}}^{d}(d-s)^{m-1} \mathrm{e}^{-\mathrm{i} s t} d s .
\end{aligned}
$$

The expression

$$
t \mapsto \sum_{j=1}^{n} d_{j}^{\ell} \prod_{\substack{k=1 \\ k \neq j}}^{n}\left(1+\frac{t}{\left(d_{j}-d_{k}\right)}\right)
$$

is a polynomial in $t$ of degree not larger than $\ell$. Indeed, it is obviously permutation symmetric in the $d_{j}$ and jointly homogeneous of degree $\ell$ in $t$ and the $d_{j}$. Replacing all the $d_{j}$ by $d_{j}+\alpha$, we obtain a polynomial in $\alpha$ of degree less than or equal to $\ell$. 
We then differentiate (6) $m$ times with respect to $t$ and put $t=0$. For $\ell<m$ we obtain

and therefore also

$$
\sum_{j=1}^{n} d_{j}^{\ell} \sum_{\substack{k_{1}, k_{2}, \ldots, k_{m} \\ k_{1}<k_{2}<\ldots<k_{m} \\ k_{\ell} \neq j}} \frac{1}{\left(d_{j}-d_{k_{1}}\right) \cdots\left(d_{j}-d_{k_{m}}\right)}=0
$$

$$
\sum_{j=1}^{n}\left(d_{j}-d_{1}\right)^{\ell} \sum_{\substack{k_{1}, k_{2}, \ldots, k_{m} \\ k_{1}<k_{2}<\cdots .<k_{m} \\ k_{\ell} \neq j}} \frac{1}{\left(d_{j}-d_{k_{1}}\right) \cdots\left(d_{j}-d_{k_{m}}\right)}=0
$$

We plug the Taylor expansion (5) in (4) and observe that, by (7), the coefficients of negative powers of $t$ all vanish. Thus, we obtain

$$
\begin{aligned}
F(\mathbf{d} ; t)= & \sum_{k=1}^{n} \mathrm{e}^{-\mathrm{i} d_{k} t}+\sum_{m=1}^{n} \frac{1}{n^{m}} \sum_{j=1}^{n} \sum_{\substack{k_{1}, k_{2}, \ldots, k_{m} \\
k_{1}<k_{2}<\ldots<k_{m} \\
k_{\ell} \neq j}} \frac{1}{(m-1) !} \\
& \times \int_{d_{1}}^{d_{j}} \mathrm{e}^{-\mathrm{i} s t} \frac{\left(d_{j}-s\right)^{m-1}}{\left(d_{j}-d_{k_{1}}\right) \cdots\left(d_{j}-d_{k_{m}}\right)} d s .
\end{aligned}
$$

We shall now rewrite this formula as

$$
F(\mathbf{d} ; t)=\sum_{k=1}^{n} \mathrm{e}^{-\mathrm{i} d_{k} t}+\sum_{m=1}^{n-1} \frac{1}{n^{m}(m-1) !} \int_{d_{1}}^{d_{n}} \mathrm{e}^{-\mathrm{i} s t} G_{m}(\mathbf{d} ; s) d s .
$$

The first term corresponds to an atomic measure attributing an equal weight to each of the eigenvalues of $H_{0}$, while the $m$-terms are the $m$-point contributions to the absolutely continuous part of the inverse Fourier transform of $F$. More precisely,

$$
G_{m}(\mathbf{d} ; s)=\sum_{\substack{\tilde{\tilde{\mathbf{d}}} \\ \tilde{\mathbf{d}} \subset \mathbf{d} \\ \#(\mathbf{d})=m+1}} S_{m}(\tilde{\mathbf{d}} ; s),
$$

where for any ordered $(m+1)$-tuple $\tilde{\mathbf{d}}=\left\{\tilde{d}_{1}, \tilde{d}_{2}, \ldots, \tilde{d}_{m+1}\right\}$,

$$
S_{m}(\tilde{\mathbf{d}} ; s):= \begin{cases}0 & \text { if } x \leq \tilde{d}_{1}, \\ \sum_{j=\ell+1}^{m+1} \frac{\left(\tilde{d}_{j}-s\right)^{m-1}}{\left(\tilde{d}_{j}-\tilde{d}_{1}\right) \cdots\left(\tilde{d}_{j}-\tilde{d}_{m+1}\right)} & \text { if } x \in\left[\tilde{d}_{\ell}, \tilde{d}_{\ell+1}\right], \\ 0 & \text { if } x \geq \tilde{d}_{m+1} .\end{cases}
$$

On $\left(\tilde{d}_{1}, \tilde{d}_{m+1}\right), S_{m}$ consists piecewise of polynomials of degree $m-1$ and it is $(m-2)$ times continuously differentiable. The function $S_{m}$ lands like $\left(\tilde{d}_{m+1}-\cdot\right)^{m-1}$ at $\tilde{d}_{m+1}$ and, by reflection symmetry, one shows that $S_{m}$ has the simple expression

$$
S_{m}(\tilde{\mathbf{d}} ; s)=\frac{\left(s-\tilde{d}_{1}\right)^{m-1}}{\left(\tilde{d}_{2}-\tilde{d}_{1}\right)\left(\tilde{d}_{3}-\tilde{d}_{1}\right) \cdots\left(\tilde{d}_{m+1}-\tilde{d}_{1}\right)}
$$

on $\left[d_{1}, d_{2}\right]$. Therefore, $S_{m}$ starts off like $\left(\cdot-\tilde{d}_{1}\right)^{m-1}$ at $\tilde{d}_{1}$. Hence, $S_{m}$ is $(m-2)$ times continuously differentiable on the real line. As

$$
\int_{\tilde{d}_{1}}^{\tilde{d}_{m+1}} S_{m}(\tilde{\mathbf{d}} ; s) d s=\frac{1}{m}
$$


the $S_{m}(\tilde{\mathbf{d}} ; \cdot)$ are precisely the well-known B-splines from approximation theory [3]. Each $\tilde{\mathbf{d}}$, together with the normalization condition (9), uniquely determines a spline $S_{m}$ of order $(m-1)$ and $S_{m}>0$ on $\left(\tilde{d}_{1}, \tilde{d}_{m+1}\right)$. If this were not true, then $S_{m}^{\prime}$ should have at least three roots in $\left(\tilde{d}_{1}, \tilde{d}_{m+1}\right)$ and the piecewise linear $(m-2)$ th derivative should have $m+1$ roots, which is impossible.

The simplest non-trivial case is the roof function $S_{2}\left(\left\{d_{1}, d_{2}, d_{3}\right\} ; \cdot\right)$, which is continuous and piecewise linear with nodes at $\left\{d_{1}, d_{2}, d_{3}\right\}$. Its explicit form is given by

$$
S_{2}\left(\left\{d_{1}, d_{2}, d_{3}\right\} ; s\right)= \begin{cases}0 & \text { if } s \leq d_{1}, \\ \frac{s-d_{1}}{\left(d_{2}-d_{1}\right)\left(d_{3}-d_{1}\right)} & \text { if } d_{1} \leq s \leq d_{2}, \\ \frac{d_{3}-s}{\left(d_{3}-d_{1}\right)\left(d_{3}-d_{2}\right)} & \text { if } d_{2} \leq s \leq d_{3}, \\ 0 & \text { if } d_{3} \leq s .\end{cases}
$$

\section{FREE NON-COMMUTATIVE RANDOM VARIABLES}

Let $\mathcal{M}$ be a type $\mathrm{II}_{1}$ von Neumann algebra with faithful normal tracial state $\tau$. Self-adjoint elements of $\mathcal{M}$ are called non-commutative random variables. Random variables $a=a^{*}, b=b^{*} \in \mathcal{M}$ are said to be in free relation if

$$
\tau\left(p_{1}(a) q_{1}(b) p_{2}(a) q_{2}(b) \ldots p_{n}(a) q_{n}(b)\right)=0
$$

whenever $p_{1}, p_{2}, \ldots, p_{n}, q_{1}, q_{2}, \ldots, q_{n}$ are polynomials such that $\tau\left(p_{i}(a)\right)=\tau\left(q_{i}(b)\right)$ $=0(1 \leq i \leq n)$. Non-commutative random variables in free relation arise from random matrix models. (For an introduction to free random variables and their random matrix model, see the book [7].) Let $H_{n}$ and $K_{n}$ be $n \times n$ random matrices for every $n \in \mathbb{N}$. They form a random matrix model for the pair $a=a^{*}, b=b^{*} \in \mathcal{M}$ if

$$
\tau_{n}\left(P\left(H_{n}, K_{n}\right)\right) \rightarrow \tau(P(a, b))
$$

for any polynomial $P$ of two non-commuting indeterminates. A very remarkable result, due to Voiculescu, tells us that if $(a, b)$ has a random matrix model such that $H_{n}$ is a Wigner matrix and $K_{n}$ is independent of $H_{n}$, then $a$ and $b$ are in free relation. This fact is a manifestation of asymptotic freeness; see [11], 12] or [7. It is a much easier fact (called Wigner theorem) that under the above conditions $a$ is a standard semicircular element, that is,

$$
\tau\left(a^{n}\right)=\frac{1}{2 \pi} \int_{-2}^{2} x^{n} \sqrt{4-x^{2}} d x \quad(n \in \mathbb{N}) .
$$

Theorem. Let $a$ and $b$ be self-adjoint operators in a von Neumann algebra with faithful normal trace $\tau$. Assume that $a$ and $b$ are in free relation with respect to $\tau$ and that $a$ is standard semicircular. Then $t \mapsto \tau(\exp (a-i t b))$ is a positive definite function: there exists a unique measure $\mu$ such that

$$
\tau(\exp (a-i t b))=\frac{1}{\sqrt{2 \pi}} \int e^{-i t x} d \mu(x) \quad(t \in \mathbb{R}) .
$$

The support of $\mu$ is contained in the convex hull of of the spectrum of $b$.

Since a pair $(a, b)$ in the theorem admits a random matrix model with Wigner matrices $H_{n}$ and non-random diagonal matrices $K_{n}$ (see [11] or Cor. 4.3.6 in [7]), the result follows from our first theorem for finite matrices. 
It was computed in [5] that in the case when both $a$ and $b$ are standard semicircular, we have

$$
\tau\left(\mathrm{e}^{a+\mathrm{i} t b}\right)=\frac{1}{2 \pi} \int_{-2}^{2} \mathrm{e}^{\mathrm{i} u t} \sinh \left(\sqrt{4-u^{2}}\right) d u .
$$

In this example the Bochner measure is explicit, however this situation is rather exceptional. We consider the example in which the spectrum of $b$ is $\{\alpha, \beta\}$ and the distribution gives equal weights $1 / 2$ at the points $\alpha<\beta$. A matrix model for this situation is obtained by choosing, for even $n, H_{0}$ diagonal with $n / 2$ eigenvalues close to $\alpha$ and the others close to $\beta$. In this case, we may take the limit $n \rightarrow \infty$ in (8) term by term. The limit of a spline function $G_{m}(\tilde{\mathbf{d}} ; s)$ when $\tilde{d}_{1}, \tilde{d}_{2}, \ldots, \tilde{d}_{k}$ tend to $\alpha$ and $\tilde{d}_{k+1}, \tilde{d}_{k+2}, \ldots, \tilde{d}_{m+1}$ to $\beta$ is easily seen to be

$$
\left(\begin{array}{c}
m-1 \\
k-1
\end{array}\right) \frac{(s-\alpha)^{m-k}(\beta-s)^{k-1}}{(\beta-\alpha)^{m}} \quad(\alpha \leq s \leq \beta)
$$

and 0 elsewhere. The limit $n \rightarrow \infty$ is now straightforward:

$$
\tau(\exp (a-\mathrm{i} t b))=\frac{1}{2}\left(\mathrm{e}^{-\mathrm{i} \alpha t}+\mathrm{e}^{-\mathrm{i} \beta t}\right)+\int_{\alpha}^{\beta} \mathrm{e}^{-\mathrm{i} s t} f(s) d s,
$$

where $f(s)$ is explicitly given by

$$
\sum_{m=0}^{\infty} \frac{1}{2^{m+2}((m+1) !)^{2}(\beta-\alpha)^{m+1}} \sum_{k=0}^{m}\left(\begin{array}{c}
m+1 \\
k
\end{array}\right)\left(\begin{array}{c}
m+1 \\
k+1
\end{array}\right)(s-\alpha)^{m-k}(\beta-s)^{k} .
$$

The Bochner measure of $t \mapsto \tau(\exp (a-\mathrm{i} t b))$ is therefore a sum of an atomic part giving equal weight to the points which support the Bernoulli variable and an absolutely continuous part with support $[\alpha, \beta]$.

\section{REFERENCES}

1. D. Bessis, P. Moussa, and M. Villani, Monotonic converging variational approximations to the functional integrals in quantum statistical mechanics, J. Math. Phys. 16 (1975), 2318-2325. MR 54:4471

2. N. Berline, E. Getzler and M. Vergne, Heat Kernels and Dirac Operators, Springer, BerlinHeidelberg-New York, 1992. MR 94e:58130

3. B.D. Bojanov, H.A. Hakopian and A.A. Sahakian, Spline Functions and Multivariate Interpolations, Kluwer, Dordrecht, 1993. MR 94k:41001

4. E. Brézin, Dyson's universality in generalized ensembles of random matrices, in The Mathematical Beauty of Physics, J.M. Drouffe and J.B. Zuber (eds.), World Scientific, 1997, pp. 111. MR 98k:82073

5. M. Fannes, D. Petz, On the function Tr $e^{H+\mathrm{i} t K}$, Int. J. Math. and Math. Sci. 29 (2002), 389-393.

6. M. Gaudin, Sur la transformée de Laplace de $\operatorname{tr} \mathrm{e}^{-A}$ considérée comme fonction de la diagonale de A, Ann. Inst. Henri Poincaré A 28 (1978), 431-442. MR 81g:44004

7. F. Hiai and D. Petz, The Semicircle Law, Free Random Variables and Entropy, Mathematical Surveys and Monographs, Vol. 77, Amer. Math. Soc., Providence, 2000. MR 2001j:46099

8. M.L. Mehta, Random Matrices, Second edition, Academic Press, Boston, 1991. MR 92f:82002

9. M.L. Mehta and K. Kumar, On an integral representation of the function $\operatorname{Tr} \exp (A-\lambda B), J$. Phys. A 9 (1976), 197-206. MR 55:2954

10. P. Moussa, On the representation of $\operatorname{Tr} \exp (A-\lambda B)$, Reviews in Math. Phys. 12 (2000), 621-655. MR 2001h:47016 
11. D. Voiculescu, Limit laws for random matrices and free products, Invent. Math. 104 (1991), 201-220. MR 92d:46163

12. D. Voiculescu, A strengthened asymptotic freeness result for random matrices with applications to free entropy, Internat. Math. Res. Notices (1998), 41-63. MR 2000d:46080

Instituut voor Theoretische Fysica, K.U. Leuven, B-3001 Leuven, Belgium

Department for Mathematical Analysis, Budapest University of Technology and Economics, H-1521 Budapest Xi., Hungary 